\title{
What Are the Clinical Features and Etiology of Eosinophilic Liver Infiltration?
}

\author{
Da Woon $\mathrm{Sim}^{1}$, Dong Jun Son ${ }^{2}$, Eunae $\mathrm{Cho}^{2}$, Sung Kyu $\mathrm{Choi}^{2}$, Sang Soo $\mathrm{Shin}^{3}$, and Chung Hwan Jun ${ }^{2}$ \\ Divisions of ${ }^{1}$ Allergy, Asthma, and Clinical Immunology and ${ }^{2}$ Gastroenterology, Department of Internal Medicine, and ${ }^{3}$ Department of \\ Radiology, Chonnam National University Medical School, Gwangju, Korea
}

Background/Aims: Although eosinophilic liver infiltration (ELI) is not rare, few data exist regarding its clinical characteristics and etiology. Therefore, we evaluated these aspects to better understand the clinical implications of this lesion type, which is reasonably common in Korea. Methods: Patients suspected of having ELI, based on abdominal computed tomography results obtained between January 2010 and September 2017, were enrolled in this retrospective study. The presumptive etiologies of ELI were categorized as parasite infections, hypereosinophilic syndrome (HES), eosinophilic granulomatosis with polyangiitis (EGPA), malignancies, and unidentified. Clinical courses and treatment responses were also evaluated. Results: The mean age of the enrolled patients (male, 237/328) was 62 years. Most patients (63\%) were diagnosed incidentally and had peripheral eosinophilia (90\%). Only $38 \%$ of the enrolled patients $(n=126)$ underwent further evaluations to elucidate the etiology of the suspected ELI; 82 (25\%) had parasite infections, 31 (9\%) had HES, five (2\%) had EGPA, and five (2\%) had drug reactions in conjunction with eosinophilia and systemic symptoms. Almost half of the other enrolled patients had cancer. Radiologic resolution was achieved in 191 patients (61\%; median time to radiologic resolution, 185 days). Resolution of peripheral eosinophilia was achieved in 220 patients (79\%). In most cases, the course of ELI was benign. Conclusions: This large ELI study is unique in that the incidence rate, underlying diseases, and clinical courses were comprehensively evaluated. Clinicians should investigate the etiology of ELI, as several of the underlying diseases require intervention rather than observation. (Gut Liver 2019;13:183-190)

Key Words: Eosinophilic infiltration; Etiology; Hypereosinophilic syndrome; Liver; Parasite

\section{INTRODUCTION}

Eosinophilic liver infiltration (ELI) can be observed on computed tomography (CT), and represents a common, focal, eosinophil-related inflammation, with or without necrosis. ${ }^{1}$ The lesions appear on CT scans as multiple, hypoattenuated, small, round lesions that have blurred margins and are mainly discerned during the portal phase. ${ }^{2}$ As a result of imaging advances, ELI is frequently found in clinical practice. ${ }^{1,3}$ Although the lesions are often found because of clinical symptoms, they are sometimes incidentally detected during routine imaging checkups in patients with cancer or during regular checkups in healthy persons. ${ }^{4}$

ELI is associated with several common conditions that include peripheral eosinophilia, ${ }^{2}$ such as parasitic infections, allergic diseases, and drug hypersensitivities. ${ }^{5}$ Although associated with peripheral eosinophilia, a previous case report suggested that ELI is not always accompanied by peripheral eosinophilia. ${ }^{6}$ Therefore, the strength of the relationship between peripheral eosinophilia and suspected ELI remains unclear.

In daily practice, we frequently encounter patients demonstrating CT findings suggestive of ELI. Until recently, ELI has tended to be regarded as having a benign course, resulting in its only being noted in imaging studies or being completely ignored. ${ }^{1}$ However, various tests should be performed to evaluate the etiology of peripheral eosinophilia and ELI.

Parasitic infections, proven using serum samples and enzymelinked immunosorbent assays (ELISAs), continue to be prevalent in Korea. ${ }^{7,8}$ Paranasal sinus (PNS) imaging, total immunoglobulin E (IgE), and allergen-specific IgE assessments can be used to evaluate a patient's atopic and allergic disease status. ${ }^{9}$ Furthermore, anti-neutrophil cytoplasmic autoantibody (ANCA) and antinuclear antibody (ANA) levels can be used in the diagnosis of connective tissue disease. ${ }^{10,11}$ Chest X-rays, vitamin $\mathrm{B}_{12}$

Correspondence to: Chung Hwan Jun (https://orcid.org/0000-0002-7136-8350)

Division of Gastroenterology, Department of Internal Medicine, Chonnam National University Hospital, 42 Jebong-ro, Dong-gu, Gwangju 61469, Korea

Tel: +82-62-220-6296, Fax: +82-62-220-6318, E-mail: estevanj@naver.com

Received on June 8, 2018. Revised on July 27, 2018. Accepted on August 9, 2018. Published online January 3, 2019

pISSN 1976-2283 eISSN 2005-1212 https://doi.org/10.5009/gnl18266

Da Woon Sim and Dong Jun Son contributed equally to this work as first authors.

@ This is an Open Access article distributed under the terms of the Creative Commons Attribution Non-Commercial License (http://creativecommons.org/licenses/by-nc/4.0) which permits unrestricted non-commercial use, distribution, and reproduction in any medium, provided the original work is properly cited. 
and tryptase levels, and liver and bone marrow biopsies can be used to evaluate hypereosinophilic syndrome (HES) and other organ involvement due to eosinophilia. ${ }^{12}$ Additionally, atypical lymphocytes in a peripheral blood smear (PBS) can provide important clues regarding the diagnosis of drug reactions with eosinophilia and systemic symptoms (DRESS) syndrome. ${ }^{13}$

Previous studies have mainly focused on the radiologic findings and clinical features associated with ELI. ${ }^{1,2,14}$ However, there are limited comprehensive data regarding the etiologies and treatment of patients with suspected ELI. ${ }^{1,2}$ Therefore, we evaluated these aspects of patients with suspected ELI.

\section{MATERIALS AND METHODS}

\section{Patients}

We retrospectively enrolled patients demonstrating suspected ELI on their abdominal CT scans, taken between January 2010 and September 2017, from the electronic medical records at Chonnam National University Hospital, South Korea.

\section{Ethical considerations}

The present study was conducted in accordance with the ethical guidelines of the Declaration of Helsinki. The study protocol was approved by the Institutional Review Board of Chonnam National University Hospital (IRB No. CNUH-2017-290). The informed consent was waived.

\section{Study design}

A complete review of the medical records was conducted. This study involved convenience sampling, and all consecutive cases were included, without a formal sample size analysis.

The following data were collected: sex, age, medical history (drug history and allergies), food ingestion history (eating raw liver or meat of cows or other animals, raw blood of animals like deer, raw freshwater fish, and raw Chinese mitten crab), PNS images, chest X-rays, other organ involvement, laboratory findings (including leukocyte count, total eosinophil count, and eosinophil percentage), PBS results, vitamin $\mathrm{B}_{12}$ level, ANCA and ANA levels, and intake of antiparasitic medications. In addition, the follow-up imaging modalities, intervals, and lesion resolution status were reviewed. The medical review was conducted by two physicians.

Patients were classified into the following groups, based on the suspected cause of the ELIs: parasite, HES, eosinophilic granulomatosis with polyangiitis (EGPA), neoplasm, DRESS syndrome, and unidentified.

\section{Definitions}

Eosinophilia is defined as eosinophil count in the peripheral blood exceeding 500 cells $/ \mathrm{mL} .{ }^{15}$ Eosinophilia has been categorized into three groups according to total eosinophil count: mild, 500 to 1,500 cells/mL; moderate, 1,500 to 5,000 cells $/ \mathrm{mL}$; severe, $>5,000$ cells $/ \mathrm{mL}^{16}$

Patients with serology test results positive for Paragonimus westermani, Clonorchis sinensis, or Toxocara canis or a positive stool examination were classified into the parasite group. The neoplasm group comprised patients with biopsy-diagnosed cancer. Patients were classified into the HES group if they fulfilled the following criteria, using the approach of Chusid et al.: ${ }^{17}$ presence of peripheral blood eosinophilia, with an absolute eosinophil count of $>1,500$ cells/mL for $>6$ months; no other evident cause of eosinophilia, including allergic diseases or parasitic infections; or the presence of signs or symptoms of organ involvement by the eosinophilic infiltrate. The EGPA group comprised patients satisfying $\geq 4$ of the following 6 American College of Rheumatology (1990) criteria: ${ }^{18}$ asthma, eosinophilia ( $>10 \%$ of the total whole blood cell count), neuropathy, nonfixed pulmonary infiltration, PNS abnormalities, and extravascular eosinophils. The DRESS syndrome group comprised patients who fulfilled the RegiSCAR criteria for DRESS syndrome. ${ }^{13}$ The RegiSCAR criteria include the presence of at least three of the following seven characteristics: skin eruptions, fever $\left(>38^{\circ} \mathrm{C}\right)$, lymphadenopathy involving at least two sites, involvement of at least one internal organ, lymphocytosis $\left(>4 \times 10^{3} / \mu \mathrm{L}\right)$ or lymphocytopenia $\left(<1.5 \times 10^{3} / \mu \mathrm{L}\right)$, blood eosinophilia $(>10 \%$ or $700 /$ $\mu \mathrm{L})$, and thrombocytopenia $\left(<120 \times 10^{3} / \mu \mathrm{L}\right)$. Patients who did not meet any of the above criteria were allocated to the unidentified group.

During the evaluation of the clinical course of the suspected ELI, based on CT results, we defined radiologic resolution as the disappearance of results on follow-up imaging.

\section{Statistical analysis}

Statistical analyses were performed using SPSS version 20.0 (IBM Corp., Chicago, IL, USA). Continous data are presented as median (range), and categorical data are shown as absolute and relative frequencies. Group differences were evaluated using the Kruskal-Wallis test followed by the Dunn test for continuous variables and Fisher exact or chi-square tests for categorical variables. $p \leq 0.05$ was considered statistically significant.

\section{RESULTS}

\section{Baseline patient characteristics}

A total of 79,739 patients underwent abdominal CT scans during the study enrollment period, including $328(0.41 \%)$ cases with suspected ELI, based on CT findings. The initial CT scans were conducted in 27 departments, mainly within the Division of Gastroenterology and the Departments of Internal Medicine and General Surgery. Table 1 shows the baseline characteristics of enrolled patients. The median age of the enrolled patients was 62 years (range, 19 to 95 years), including 237 (72\%) men (Fig. 1A). A total of 288 patients (90\%) had peripheral eosinophilia, with a median peripheral eosinophil count of $1,033 / \mu \mathrm{L}$ (range, 0 
Table 1. Baseline Characteristics According to Underlying Disease

\begin{tabular}{|c|c|c|c|c|c|c|c|}
\hline Characteristic & $\begin{array}{l}\text { Parasite } \\
(n=82)\end{array}$ & $\begin{array}{l}\text { HES } \\
(n=31)\end{array}$ & $\begin{array}{l}\text { EGPA } \\
(n=5)\end{array}$ & $\begin{array}{l}\text { DRESS syndrome } \\
\qquad(\mathrm{n}=5)\end{array}$ & $\begin{array}{l}\text { Neoplasm } \\
(\mathrm{n}=2)\end{array}$ & $\begin{array}{l}\text { Unidentified } \\
\qquad(\mathrm{n}=203)\end{array}$ & $\mathrm{p}$-value \\
\hline Age, yr* & $60(19-82)$ & 67 (48-88) & $63(32-81)$ & $52(48-56)$ & $52(42-62)$ & $63(20-95)$ & 0.084 \\
\hline Elderly $^{\dagger}$ & $34(42)$ & $18(58)$ & $2(40)$ & 0 & 0 & $95(47)$ & 0.348 \\
\hline Male sex & $63(77)$ & $24(78)$ & $3(60)$ & $2(40)$ & $1(50)$ & $142(70)$ & 0.581 \\
\hline \multirow[t]{2}{*}{ WBC, cells $/ \mu \mathrm{L}$} & 8,400 & 9,700 & 11,900 & 11,300 & 6,150 & 7,000 & $<0.0001$ \\
\hline & $(4,700-17,700)$ & $(5,800-24,600)$ & $(6,200-30,900)$ & $(5,300-24,300)$ & $(6,100-6,200)$ & $(620-29,100)$ & \\
\hline Leukocytosis & $8(10)$ & $10(32)$ & $2(40)$ & $3(60)$ & 0 & $12(6)$ & $<0.0001$ \\
\hline \multirow[t]{2}{*}{ TEC, cells/mL } & 1,545 & 2,930 & 1,800 & 1,800 & 295 & 727 & $<0.0001$ \\
\hline & $(0-11,400)$ & $(850-15,360)$ & $(1,000-21,870)$ & $(320-3,500)$ & $(230-360)$ & $(0-7,160)$ & \\
\hline Eosinophilia & 81 (99) & $31(100)$ & $5(100)$ & $5(100)$ & $1(50)$ & $168(83)$ & $<0.0001$ \\
\hline Cancer history & $16(20)$ & $3(10)$ & 0 & 0 & $2(100)$ & 93 (46) & $<0.0001$ \\
\hline Raw food ingestion & $56(68)$ & $20(65)$ & $2(40)$ & 0 & 0 & $13(6)$ & $<0.0001$ \\
\hline Radiologic resolution $^{\ddagger}$ & $59(72)$ & $15(48)$ & $2(40)$ & $1(20)$ & 0 & $114(56)$ & 0.007 \\
\hline
\end{tabular}

Data are presented as number (\%) or median (range).

HES, hypereosinophilic syndrome; EGPA, eosinophilic granulomatosis with polyangiitis; DRESS, drug reactions with eosinophilia and systemic symptoms; WBC, white blood cell; TEC, total eosinophil count.

*Mean (range); ${ }^{\dagger}$ Elderly was defined as age greater than 65 years; ${ }^{\ddagger}$ Radiologic follow-up was performed for 227 of the study populations.

A

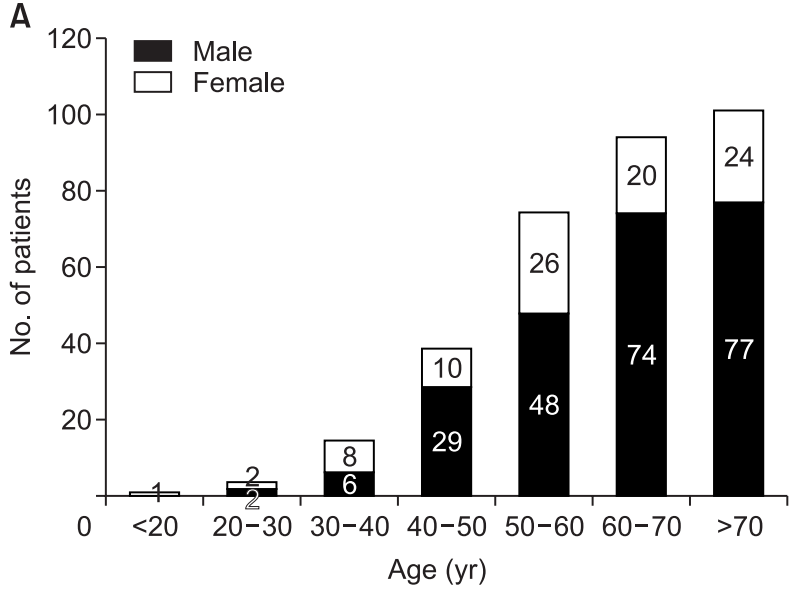

B

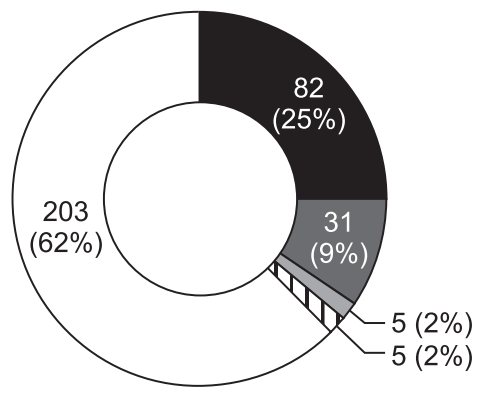

Fig. 1. (A) Distribution of gender and age in enrolled patients. (B) Presumptive causes of eosinophilic liver infiltration in this study.

to $21,870 / \mu \mathrm{L}$ ). The whole blood cell and total eosinophil counts differed significantly among the six etiologic groups. In the neoplasm and unidentified groups, the mean total eosinophil counts were significantly lower than those in the other groups $(\mathrm{p}<0.001)$.

Thirty-one patients (9\%) fulfilled the HES criteria, with more than half of these patients showing an involvement of $>2$ organs (data not shown). Other than the liver, the next most commonly involved organ was the lung.

Five patients (1.5\%) fulfilled the EGPA criteria and five (1.5\%) fulfilled the DRESS syndrome criteria. Among patients with DRESS syndrome, the culprit drugs were identified as allopurinol (two patients, 40\%), carbamazepine (two, 40\%), and tuberculosis medications (one, 20\%) (data not shown).

Patients in the unidentified group were further divided into two subgroups, based on the presence of cancer (data not shown). The identified malignancies in patients with cancer were stomach cancer $(38,41 \%)$, colorectal cancer $(26,28 \%)$, cholangiocarcinoma (seven, 8\%), esophageal cancer (six, 7\%), and other cancers including hepatocellular carcinoma, lymphoma, renal cell carcinoma, lung cancer, prostate cancer, cervix cancer, and breast cancer. A total of 110 patients (33.5\%) in the unidentified etiology group did not have cancer. Within the unidentified group, a significantly higher proportion of patients with cancer had peripheral eosinophilia than did patients without cancer ( $\mathrm{p}=0.010)$ (data not shown). However, in terms of leukocytosis, the patients with cancer did not differ from those without cancer.

A total of 10 patients (3\%) with suspected ELI underwent liver biopsies (data not shown). Of those, only two patients had 
confirmed metastatic malignancies. Although the other eight patients did not have confirmed metastatic malignancies, they had diverse results, including mild inflammation in the portal areas (six patients), a cirrhotic nodule (one patient with underlying liver cirrhosis), and liver involvement of HES (one patient).

\section{Testing patterns for patients with suspected ELI}

Table 2 shows the diagnostic tests used to evaluate ELI in the enrolled patients. In most cases, clinicians conducted tests for whole blood cell and total eosinophil counts. However, further evaluations to elucidate the cause of the peripheral eosinophilia were infrequently conducted; only 126 patients underwent at least one additional test for this purpose. The specific tests and numbers of patients tested were as follows (Table 2): PBS (126 patients, 38\%), IgE (108, 33\%), allergen sensitization profile (35, $11 \%)$, simple chest X-ray (287, 88\%), PNS imaging (47, 14\%), vitamin $\mathrm{B}_{12}$ level $(123,38 \%)$, ELISA for $P$. westermani, C. sinensis, and $T$. canis $(126,38 \% ; 130,40 \% ; 111,34 \%$, respectively), and stool examination (88, 27\%). Bone marrow biopsies were performed in 27 patients (8\%), with 30\% demonstrating abnormal results. Abnormal chest X-ray or chest CT findings were observed in 70 patients (24\%). Physicians collected raw food histories from only 100 patients (30\%); of those, 12 (12\%) did not undergo any examinations for parasitic infections.

\section{Presumptive causes of the suspected eosinophil liver infiltration}

The presumptive causes of the suspected ELI cases, based on clinical laboratory tests, other imaging, and medical histories are shown in Table 1 and Fig. 1B.

Within the parasite group, the identified parasites were $P$. westermani (four patients, 5\%), C. sinensis (14, 17\%), and $T$. canis $(64,78 \%)$; toxocariasis was the most common cause of parasitic infection. Three patients showed negative parasite antibody ELISA results in serum, but were diagnosed with a parasitic infection based on stool test results; all three had C. sinensis infections (data not shown). In a subgroup analysis, patients infected with $T$. canis tended to be older than those infected with the other two parasites. Additionally, patients infected with $T$. canis tended to have higher total eosinophil counts than did patients infected with the other parasites. However, neither of these results demonstrated statistical significance (Table 3).

\section{ELI resolution and response to antiparasitic treatments}

A total of 126 patients were treated with antiparasitic drugs, regardless of parasite examination results (Table 4); of these, 74 (59\%) were determined to have parasite infections. The empiric parasitic treatment involved oral albendazole (400 mg, twice/ day, for 1 week) and/or oral praziquantel $(25 \mathrm{mg} / \mathrm{kg}$, three

Table 2. Clinical Approach for the Evaluation of the Etiology of Eosinophilic Liver Infiltration ( $\mathrm{n}=328)$

\begin{tabular}{lclc}
\hline \multicolumn{1}{c}{ Diagnostic test } & No. of patients tested & \multicolumn{1}{c}{ Abnormal results } & $\begin{array}{c}\text { No. of patients with } \\
\text { abnormal results }\end{array}$ \\
\hline Whole blood cell count & $324(99)$ & Leukocytosis & $35(11)$ \\
Eosinophil count & $324(99)$ & Eosinophilia & $291(90)$ \\
PBS & $126(38)$ & Abnormal result of PBS & $120(94)$ \\
Total IgE & $108(33)$ & Elevation of total IgE & $35(11)$ \\
Allergen specific IgE & $35(11)$ & Sensitization of one or more allergen & $13(37)$ \\
Chest X-ray & $287(88)$ & Abnormal finding of chest X-ray & $70(24)$ \\
PNS X-ray & $47(14)$ & Abnormal finding of PNS X-ray & $19(40)$ \\
Vitamin B ${ }_{12}$ & $123(38)$ & Elevation of vitamin B & $23(19)$ \\
Bone marrow biopsy & $27(8)$ & Abnormal finding of bone marrow biopsy & $7(26)$ \\
ANCA & $68(21)$ & Abnormal result of ANCA & 0 \\
ANA & $63(19)$ & Abnormal result of ANA & $5(8)$ \\
Tryptase & $65(20)$ & Abnormal result of tryptase & $1(2)$ \\
Parasite test & & & Positive result \\
ELISA for Paragonimus westermani & $126(38)$ & Positive result & $4(3)$ \\
ELISA for Clonorchis sinensis & $130(40)$ & Positive result & $14(11)$ \\
ELISA for Toxocara canis & $111(34)$ & Positive result & $64(58)$ \\
Stool test for parasite & $88(27)$ & Ingestion of raw foods & $5(6)$ \\
History taking of raw foods & $100(30)$ & & $91(91)$ \\
\hline are & & &
\end{tabular}

Data are presented as number $(\%)$.

PBS, peripheral blood smear; IgE, immunoglobulin E; PNS, paranasal sinus series; ANCA, anti-neutrophil cytoplasmic autoantibody; ANA, antinuclear antibody; ELISA, enzyme-linked immunosorbent assay. 
Table 3. Clinical Characteristics of Parasitic Infection by Presumptive Causes ( $\mathrm{n}=82)$

\begin{tabular}{|c|c|c|c|c|}
\hline Characteristic & Paragonimus westermani & Clonorchis sinensis & Toxocara canis & p-value \\
\hline No. of patients & $4(5)$ & $14(17)$ & $64(78)$ & \\
\hline Male sex & $2(50)$ & $10(71)$ & $51(80)$ & 0.261 \\
\hline Age, $\mathrm{yr}^{*}$ & $56(46-64)$ & 59 (19-78) & $64(27-82)$ & 0.301 \\
\hline WBC, cells $/ \mathrm{mL}$ & $9,650(6,600-14,500)$ & $9,550(5,600-17,700)$ & $7,850(4,700-17,300)$ & 0.126 \\
\hline TEC, cells/mL & $3,180(1,300-7,395)$ & $1,731(600-11,400)$ & $1,400(0-8,510)$ & 0.055 \\
\hline Eosinophilia & $4(100)$ & $14(100)$ & $63(98)$ & 1.000 \\
\hline Moderate eosinophilia & $3(75)$ & $10(71)$ & $30(47)$ & 0.201 \\
\hline Radiologic resolution $^{\dagger}$ & $3(75)$ & $9(100)$ & $47(92)$ & 0.397 \\
\hline Time to radiologic resolution, day ${ }^{\dagger}$ & $188(98-387)$ & $189(52-521)$ & $183(14-1,506)$ & 0.920 \\
\hline Eosinophil normalization $^{\ddagger}$ & $2(67)$ & $12(92)$ & $56(93)$ & 0.272 \\
\hline Time to eosinophil normalization, day ${ }^{\dagger}$ & $195(42-276)$ & $93(34-196)$ & $118(2-1,323)$ & 0.617 \\
\hline
\end{tabular}

Data are presented as number (\%) or median (range).

WBC, white blood cell; TEC, total eosinophil count.

*Mean (range); ${ }^{\dagger}$ Radiologic follow-up was performed for 60 of the study populations; ${ }^{\ddagger}$ Total eosinophil count follow-up was performed for 77 of the study populations.

Table 4. Clinical Course of Suspected Eosinophil Infiltration on Liver According to the Administration of Antiparasitic Treatment

\begin{tabular}{lccc}
\hline & Untreated & Treated & p-value \\
\hline No. of patients & 202 & 126 & \\
Radiologic resolution & $114(56)$ & $77(61)$ & 0.284 \\
$\begin{array}{l}\text { Time to radiologic } \\
\text { resolution, day }\end{array}$ & $183(14-1,346)$ & $189(14-1,506)$ & 0.449 \\
\hline
\end{tabular}

Data are presented as number (\%) or median (range).

times/day, for 3 days). ${ }^{1,19}$ Although, the parasite group showed a significantly higher radiologic resolution rate than did the other groups ( $p=0.007$ ) (Table 1), there were no differences in the radiologic resolution and eosinophil normalization rates between patients empirically treated and not treated with antiparasitic drugs (56\% vs 61\%, $\mathrm{p}=0.284$ ) (Table 4). Among patients achieving radiologic resolution, the median time to resolution did not differ significantly between patients empirically treated and not treated with antiparasitic drugs $(p=0.449)$ (Table 4). Among the patients without any serum or stool evidence of parasitic infection, 50 (20\%) received antiparasitic medication and 196 (80\%) did not; there was no significant difference in the radiologic resolution rates (44\% vs 56\%) (data not shown).

\section{Clinical course and follow-up modalities of ELI}

Most patients (312 patients, 95\%) revisited the hospital after being diagnosed with suspected ELI (Table 5). More than 70\% of these patients underwent follow-up imaging, including abdominal CT (132 patients, 58\%), abdominal ultrasonography (19, $8 \%$ ), or magnetic resonance (MR) imaging (10, 4\%); 66 patients (29\%) received follow-up imaging involving more than two modalities, and most of them (53 patients, 80\%) underwent MR imaging alone or combined CT and MR imaging for follow-up
Table 5. Clinical Course and Follow-up Modalities of ELI ( $\mathrm{n}=328)$

\begin{tabular}{lc}
\hline & No. of patients \\
\hline Follow-up the clinic & $312(95)$ \\
Radiologic resolution & $191(61)$ \\
Remained the ELI & $40(13)$ \\
Time of radiologic resolution, day & $185(14-1,506)$ \\
Peripheral eosinophilia normalization & $220(67)$ \\
Remained the peripheral eosinophilia & $26(8)$ \\
Follow-up the imaging & $227(69)$ \\
Kind of follow up imaging modalities & \\
Abdominal computed tomography & $132(58)$ \\
Abdominal ultrasonography & $19(8)$ \\
Magnetic resonance imaging & $10(4)$ \\
More than 2 combined modalities use & $66(29)$ \\
Including magnetic resonance imaging & $53(80)$ \\
\hline
\end{tabular}

Data are presented as number (\%) or median (range).

ELI, eosinophilic liver infiltration.

and further evaluation (Table 5). Among the 312 patients who revisited the hospital, radiologic resolution was achieved by 191 (61\%), with a median time to resolution of 185 days (range, 14 to 1,506 days) (Table 5). The remaining 40 patients (13\%) did not achieve radiologic resolution, but peripheral eosinophilia resolution was achieved in 21 of these patients (53\%) (data not shown). The patients without radiologic resolution had a median follow-up duration of 174 days (range, 6 to 3,642 days) (data not shown).

Of the 312 patients returning to the hospital, peripheral eosinophilia was initially detected in laboratory samples from 278 patients. Among these individuals, 220 (79\%) achieved complete resolution, including normalized peripheral eosinophil 
counts; 26 (9\%) did not achieve normalized peripheral eosinophil counts; and 32 (12\%) did not undergo subsequent testing for peripheral eosinophilia.

\section{DISCUSSION}

This large, retrospective study is unique in that the incidence rate, underlying disease, and clinical course of ELI were comprehensively evaluated in the patients. In the present study, the incidence rate of clinically-diagnosed eosinophilic liver lesions was $0.41 \%$, and most patients had no specific symptoms; this is similar to a previous study, ${ }^{1}$ which reported an incidence rate of $0.68 \%$. Only 38\% of the enrolled patients underwent further evaluations to elucidate the etiology of the suspected ELI, and the most common cause was determined to be parasitic infection. The rate of radiologic resolution was $61 \%$, and the rate of peripheral eosinophilia resolution was $79 \%$. In most cases, the lesions followed a benign course. Furthermore, there was no difference in the radiologic resolution and eosinophil normalization rates between patients empirically treated and not treated with antiparasitic drugs.

The imaging and clinical features associated with ELI have been reported by many Korean researchers. ${ }^{1,2,14,20,21}$ Most recent studies have mainly focused on the radiologic findings and clinical features associated with ELI. ${ }^{1,214}$ In contrast, the present study focused on the diagnostic approach used to determine the etiology of suspected lesions.

Consistent with other studies, ${ }^{1,2} 63.1 \%$ of patients with radiologically suspected ELI did not have any specific symptoms. Further, only $38 \%$ of the patients underwent at least one test to identify the cause. Most often, physicians did not take raw food and/or drug histories to determine the likelihood of parasitic infections, which are frequent in Korea. In general, the most common causes of ELI are parasitic infections and medications; the lesions are also associated with malignant tumors. ${ }^{22}$ However, in the present study, parasitic infections and HES were identified as likely causes more frequently than expected, whereas medication-related ELI was identified less frequently than expected. HES and parasitic infections do not naturally resolve without intervention. Therefore, clinicians should perform appropriate tests and take complete histories to determine the etiology of presumptive ELI.

In Korea, toxocariasis is the most common cause of peripheral blood eosinophilia and eosinophilic infiltrations in various organs. ${ }^{12,23}$ This is consistent with our finding that $T$. canis was the most common parasite identified in patients with ELI in the present study. As in a previous study, ${ }^{24}$ more than half of the patients (41 patients, 64\%) with parasitic infections did not present with clinical symptoms such as cough, dyspnea, chest discomfort, or pruritus in the present study.

Most of the patients $(288,89 \%)$ in this study showed peripheral eosinophilia, which was not further evaluated. Regardless, approximately $10 \%$ of patients with suspected ELI were finally diagnosed with HES. This is consistent with a previous report that revealed that liver involvement is a relatively common manifestation in patients with HES. ${ }^{25}$ As this disease is a myeloproliferative disorder with persistent eosinophilia and multiple organ damage, ${ }^{26}$ it can be life-threatening; in 1989, the 5-year survival rate was $80 \%{ }^{27}$ Fortunately, HES survival rates appear to have been improving, possibly because of new medication options and quicker diagnoses. ${ }^{28}$ In the present study, all patients diagnosed with HES received treatment, and mortality was not observed. Since the early diagnosis of HES is very important in reducing patient mortality, we suggest that incidental ELI, with more than moderate peripheral eosinophilia $(>1,500$ cells $/ \mathrm{mL}$ ), may be an indicator of HES. Thus, when a patient demonstrates these two conditions (ELI CT finding and moderate eosinophilia), physicians should consider the possibility of HES. In addition, patients with suspected eosinophilic infiltration on abdominal CT images should undergo whole blood and total eosinophil count determinations, at a minimum.

Liver involvement in patients with EGPA has been rarely reported. ${ }^{29-31}$ Nevertheless, EGPA was diagnosed in 1.5\% of the patients in the present study. Although EGPA is rare, patients with ELI and a history of asthma should be questioned to determine if they fulfill the EGPA criteria. ${ }^{29}$

Liver impairment is the most common visceral manifestation of DRESS syndrome, but in most cases, it is only discovered through abnormal laboratory results. The most important treatment for medication-induced DRESS syndrome is discontinuation of the medication, as soon as possible. Thus, physicians should determine the recent medication history in patients with ELI. $^{32}$

Toxocariasis has been suggested to be the most important cause of ELI. ${ }^{23,33,34}$ As a result, one previous study suggested that empiric antiparasitic medication therapy produces more rapid radiologic resolution in patients with ELI of unidentified etiology than any other treatment. ${ }^{1}$ In Korea, a common cultural practice involves the eating of raw food, which might increase the prevalence of ELI, compared with other countries. Therefore, empirically administering anti-parasite medications to patients with suspected ELI would be appropriate in Korea. However, in this large present study, we did not observe any differences in the resolution rates between patients empirically treated or not treated with anti-parasite medications. Therefore, more studies on the effects of this treatment approach will be needed.

In our study, many patients had coexisting malignancy, but very few patients needed biopsy to distinguish ELI from metastatic malignancy and primary malignancy lesion. The reason was that some patients underwent MR imaging after abdominal CT scan. According to previous reports, MR imaging was helpful in differentiating them from the features of focal liver metastasis. ${ }^{2,35}$ Malignancy has some unique features on MR imaging. For example, malignancy showed a prominent washout of con- 
trast material in the lesions with delayed capsular enhancement, but most of the ELIs did not show a prominent washout on the dynamic delayed phase. ${ }^{22}$ MR imaging is considered a good modality for distinguishing ELI from malignancy.

Follow-up imaging showed that cases of suspected ELI pursue rather benign courses. However, ELI, like other medical conditions, needs identification of the cause and appropriate treatment. Furthermore, in the case of patients with remaining hepatic lesions during follow-up imaging, physicians should try to actively look for the etiology of ELI such as HES, drug allergy, parasite infestation, and malignancy. In cases of malignancy, the results of our study suggest that the physician may suspect hidden gastrointestinal cancer.

The present study has several limitations. First, its retrospective design is associated with known risks of bias and data limitations; thus, the duration of cases of suspected ELI could not be accurately determined. Second, the study was conducted in a tertiary hospital in Korea, and patients were preferentially enrolled if they specifically required abdominal CT scans; thus, the findings may not reflect the actual incidence of focal eosinophilic infiltrations, which are usually asymptomatic.

In conclusion, to the best of our knowledge, the present study is the largest comprehensive study to evaluate the incidence, etiology, and clinical course of suspected ELI. Although ELI is usually a benign condition commonly caused by parasitic infections, HES and malignancies are the more common causes. Therefore, physicians should undertake evaluations to establish the cause of focal hepatic eosinophil infiltrations observed in patients.

\section{CONFLICTS OF INTEREST}

No potential conflict of interest relevant to this article was reported.

\section{ACKNOWLEDGEMENTS}

This study was supported by grants (CRI 18091-1, CRI 170271) from the Chonnam National University Hospital Biomedical Research Institute.

Author contributions: D.W.S. and D.J.S. drafted the manuscript. D.J.S., E.C., S.K.C., and S.S.S. were involved in the data acquisition. D.W.S. and C.H.J. analyzed and interpreted the data, designed the research, and reviewed the manuscript.

\section{REFERENCES}

1. Kwon JW, Kim TW, Kim KM, et al. Clinical features of clinically diagnosed eosinophilic liver abscesses. Hepatol Int 2011;5:949954.

2. Yoo SY, Han JK, Kim YH, Kim TK, Choi BI, Han MC. Focal eosinophilic infiltration in the liver: radiologic findings and clinical course. Abdom Imaging 2003;28:326-332.

3. Chung YE, Kim MJ, Kim YE, Park MS, Choi JY, Kim KW. Characterization of incidental liver lesions: comparison of multidetector CT versus Gd-EOB-DTPA-enhanced MR imaging. PLoS One 2013;8:e66141.

4. Algarni AA, Alshuhri AH, Alonazi MM, Mourad MM, Bramhall SR. Focal liver lesions found incidentally. World J Hepatol 2016;8:446-451.

5. Bain BJ. Hypereosinophilia. Curr Opin Hematol 2000;7:21-25.

6. Jang IY, Yang YJ, Cho HJ, et al. Eosinophilic organ infiltration without eosinophilia or direct parasite infection. Korean J Intern Med 2014;29:126-129.

7. Choi SC, Lee SY, Song HO, Ryu JS, Ahn MH. Parasitic infections based on 320 clinical samples submitted to Hanyang University, Korea (2004-2011). Korean J Parasitol 2014;52:215-220.

8. Lee MK, Hong SJ, Kim HR. Seroprevalence of tissue invading parasitic infections diagnosed by ELISA in Korea. J Korean Med Sci 2010;25:1272-1276.

9. Gergen PJ, Arbes SJ Jr, Calatroni A, Mitchell HE, Zeldin DC. Total IgE levels and asthma prevalence in the US population: results from the National Health and Nutrition Examination Survey 2005-2006. J Allergy Clin Immunol 2009;124:447-453.

10. Sen D, Isenberg DA. Antineutrophil cytoplasmic autoantibodies in systemic lupus erythematosus. Lupus 2003;12:651-658.

11. Habash-Bseiso DE, Yale SH, Glurich I, Goldberg JW. Serologic testing in connective tissue diseases. Clin Med Res 2005;3:190193.

12. Choi D, Lim JH, Choi DC, et al. Transmission of Toxocara canis via ingestion of raw cow liver: a cross-sectional study in healthy adults. Korean J Parasitol 2012;50:23-27.

13. Kim DH, Koh YI. Comparison of diagnostic criteria and determination of prognostic factors for drug reaction with eosinophilia and systemic symptoms syndrome. Allergy Asthma Immunol Res 2014;6:216-221.

14. Jang HJ, Lee WJ, Lee SJ, Kim SH, Lim HK, Lim JH. Focal eosinophilic necrosis of the liver in patients with underlying gastric or colorectal cancer: CT differentiation from metastasis. Korean J Radiol 2002;3:240-244.

15. Osgood EE, Brownlee IE, Osgood MW, Ellis DM, Cohen W. Total differential and absolute leukocyte counts and sedimentation rates: determined for healthy persons nineteen years of age and over. Arch Intern Med 1939;64:105-120.

16. Weller PF. The immunobiology of eosinophils. N Engl J Med 1991;324:1110-1118.

17. Chusid MJ, Dale DC, West BC, Wolff SM. The hypereosinophilic syndrome: analysis of fourteen cases with review of the literature. Medicine (Baltimore) 1975;54:1-27.

18. Gioffredi A, Maritati F, Oliva E, Buzio C. Eosinophilic granulomatosis with polyangiitis: an overview. Front Immunol 2014;5:549.

19. Insiripong S, Siriyakorn N. Treatment of eosinophilia with albendazole. Southeast Asian J Trop Med Public Health 2008;39:517520. 
20. Lim JH, Lee KS. Eosinophilic infiltration in Korea: idiopathic? Korean J Radiol 2006;7:4-6.

21. Byun JH, Yang DH, Yoon SE, et al. Contrast-enhancing hepatic eosinophilic abscess during the hepatic arterial phase: a mimic of hepatocellular carcinoma. AJR Am J Roentgenol 2006;186:168173.

22. Kim YK, Kim CS, Moon WS, Cho BH, Lee SY, Lee JM. MRI findings of focal eosinophilic liver diseases. AJR Am J Roentgenol 2005;184:1541-1548.

23. Kwon NH, Oh MJ, Lee SP, Lee BJ, Choi DC. The prevalence and diagnostic value of toxocariasis in unknown eosinophilia. Ann Hematol 2006;85:233-238.

24. Lee JY, Yang MH, Hwang JH, et al. The prevalence of toxocariasis and diagnostic value of serologic tests in asymptomatic Korean adults. Allergy Asthma Immunol Res 2015;7:467-475.

25. Weller PF, Bubley GJ. The idiopathic hypereosinophilic syndrome. Blood 1994;83:2759-2779.

26. Noh HR, Magpantay GG. Hypereosinophilic syndrome. Allergy Asthma Proc 2017;38:78-81.

27. Lefebvre C, Bletry O, Degoulet P, et al. Prognostic factors of hypereosinophilic syndrome: study of 40 cases. Ann Med Interne (Paris) 1989;140:253-257.

28. Sheikh J, Weller PF. Clinical overview of hypereosinophilic syndromes. Immunol Allergy Clin North Am 2007;27:333-355.
29. Masi AT, Hunder GG, Lie JT, et al. The American College of Rheumatology 1990 criteria for the classification of Churg-Strauss syndrome (allergic granulomatosis and angiitis). Arthritis Rheum 1990;33:1094-1100.

30. Yüksel I, Ataseven H, Başar 0, et al. Churg-Strauss syndrome associated with acalculous cholecystitis and liver involvement. Acta Gastroenterol Belg 2008;71:330-332.

31. Kim HS, Jeong HK, Kim DH, Yu J, Kim Y, Koh YI. A case of churg-strauss syndrome: evidence of eosinophilic vasculitis on liver biopsy. Allergy Asthma Respir Dis 2013;1:164-167.

32. Lin IC, Yang HC, Strong C, et al. Liver injury in patients with DRESS: a clinical study of 72 cases. J Am Acad Dermatol 2015;72:984-991.

33. Musso C, Castelo JS, Tsanaclis AM, Pereira FE. Prevalence of Toxocara-induced liver granulomas, detected by immunohistochemistry, in a series of autopsies at a Children's Reference Hospital in Vitoria, ES, Brazil. Virchows Arch 2007;450:411-417.

34. Yoon YS, Lee CH, Kang YA, et al. Impact of toxocariasis in patients with unexplained patchy pulmonary infiltrate in Korea. J Korean Med Sci 2009;24:40-45.

35. Guo BL, Hu QG, Ouyang FS, et al. CT and MRI findings in focal eosinophilic infiltration of the liver. Abdom Radiol (NY) 2017;42:2874-2881. 